\title{
Orientation of adsorbed polymer in response to shear flow
}

\author{
Peter Frantz, David Perry, Steve Granick* \\ Department of Materials Science and Engineering, University of Illinois, Urbana, IL 61801, USA
}

(Received 29 April 1993; accepted 4 August 1993)

\begin{abstract}
We report preliminary evidence of shear-induced alignment of polyisoprene adsorbed from $\mathrm{CCl}_{4}$ solution on to a single solid surface. The experimental technique was Fourier transform infrared spectroscopy, in the mode of attenuated total reflection. The selective augmentation of vibrational modes oriented preferentially along the chain backbone implied chain orientation in the direction of flow. This occurred at low shear rates (about $1 \mathrm{~s}^{-1}$ ). The extent of orientation increased with increasing molecular weight, and did not depend on shear rate within the narrow range $\left(0.4-1.6 \mathrm{~s}^{-1}\right)$ available to us. After cessation of flow, relaxation toward the conformation in the quiescent state was slow.
\end{abstract}

Key words: Orientation; Polyisoprene; Shear flow

The majority of the analysis of polymer adsorption has focused on quiescent systems [1-4]: adsorption from a static solution. Despite its obvious utility as a model system, we believe the quiescent state to be a special case in both nature and technology. Polymer adsorption in the presence of a flow field occurs in such applications as biocompatibility, filtration, enhanced oil recovery, viscometry, lubrication, wastewater treatment, and manufacture of materials. In order to understand fully the surface and rheological properties of polymer layers in these situations, it is essential to understand the parameters that affect conformational changes near the surface.

Previous experimental investigations of flowinduced deformation have employed either ellipsometry [5-8] or hydrodynamic flow [9-12]. The ellipsometric technique provides a direct optical measure of the root-mean-square thickness of the adsorbed layer. The hydrodynamic flow technique measures the resistance to flow, through a porous

*Corresponding author. medium or capillary tube, due to constriction caused by the adsorbed layer. Reduction in diameter of the flow channel may be expressed as an effective hydrodynamic thickness (EHT). The literature of experimental and theoretical results has been summarized elsewhere [9]. It is immediately apparent that, depending upon the technique employed, experimental measurements of flowinduced changes in the EHT vary not only in magnitude, but in sign. Although Tirrell and co-workers [12] have shed some light on possible origins of these differences, it is clear that a basic understanding of surface properties demands direct knowledge of surface molecular orientation.

In this paper, we describe preliminary spectroscopic evidence of alignment of adsorbed polyisoprene in the presence of a shear field. Infrared spectroscopy in the mode of attenuated total reflection (ATR) was employed to measure adsorption onto a native oxide surface. The cylindrical ATR prism used was Amtir, an alloy of germanium, arsenic and selenium. Methods described previously were used to collect the IR spectra [13] 
and to prepare the surface of the ATR prism [14]. Polyisoprene (PI) standards of various degrees of polymerization were obtained from Polymer Laboratories. They were generally used as received; however, control experiments showed the same effects when the samples were precipitated from dilute solution to remove antioxidant. Characteristics (weight-average molecular weight $M_{\mathrm{w}}$, ratio of $M_{\mathrm{w}}$ to number-average molecular weight $M_{n}$ ) of the polymer samples are listed in Table 1.

The samples were dissolved in carbon tetrachloride (Fisher) at concentrations of about $1 \mathrm{mg} \mathrm{ml}^{-1}$. The net segment-surface interaction energy is expected to be close to its value, i.e. approximately $3 k T$ [15], evaluated by us using the method of Cohen Stuart et al. [16] on a chemically similar surface, silicon oxide. Experiments were performed at room temperature.

To infer orientational information from the spectra, two sets of vibrational modes were monitored. In the region of carbon-hydrogen stretching modes (approximately $2930 \mathrm{~cm}^{-1}$; region 1), no change in the integrated intensity with flow was found (see below). Although changes with flow of subpeaks were qualitatively evident from inspection of the spectra, the peaks overlapped too extensively to permit reliable separation. We surmise that the integrated intensity of the component modes had no net orientation with respect to the chain backbone [17]. However, the region of bending, twisting and wagging modes (around $1370 \mathrm{~cm}^{-1}$; region 2) complements the stretching modes, but with a significant component of the net dipole transition moment parallel to the chain

Table 1

Characteristics of the polymers studied ${ }^{\mathrm{a}}$

\begin{tabular}{rl}
\hline$M_{\mathrm{w}}{ }^{\mathrm{a}}$ & $M_{\mathrm{w}} / M_{\mathrm{n}}$ \\
\hline 60000 & 1.04 \\
135000 & 1.04 \\
460000 & 1.06 \\
1600000 & 1.08 \\
\hline
\end{tabular}

a Manufacturer's data (Polymer Laboratories, Amherst, MA). backbone [18]. Changes in the respective integrated intensities in regions 1 and 2 thus reflected overall changes in backbone orientation with respect to the (constant) electric field of the sampling beam. A similar technique was developed by Flournoy and Schaffers [19] and was recently applied to the study of adsorbed polymer in quiescent systems by van Alsten [20] and Sung [21].

In Fig. 1, the integrated intensities of the vibrations in regions 1 and 2 are plotted as a function of time for PI (molecular weight $M=$ 60000 ). Each experiment began with a stagnation period, in this case $6 \mathrm{~h}$, to allow for the slow conformational equilibration that was observed in our earlier work [22]. A shear flow of $1 \mathrm{~s}^{-1}$ was initiated after the stagnation period, and continued for several hours. At this point, the peaks in region 2 began a dramatic augmentation in intensity. Meanwhile, peaks in region 1 kept roughly the same intensity. Control experiments, in which pure $\mathrm{CCl}_{4}$ (rather than polymer solution) flowed past the adsorbed layer, gave similar findings, showing that the augmentation of peak intensity in region 2 did not reflect additional mass adsorbed.

Our tentative interpretation of the changes in oscillator strength is simple. As the flow field was introduced the dipole transition moments of the

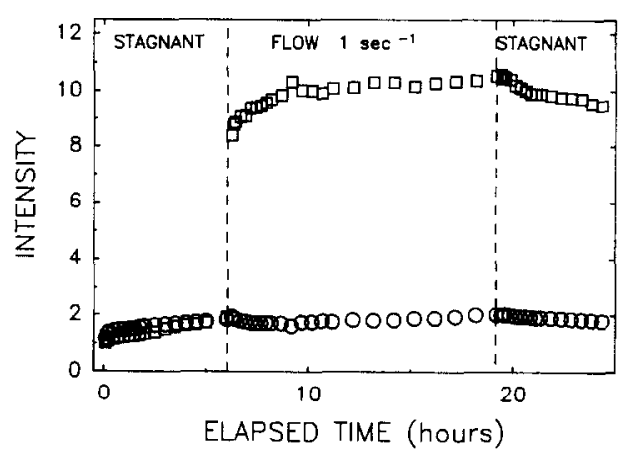

Fig. 1. Integrated intensity of polyisoprene $\left(M_{\mathrm{w}}=460000\right)$ plotted against elapsed time during adsorption from carbon tetrachloride solution (about $1 \mathrm{mg} \mathrm{ml}^{-1}$ ) onto initially bare Amtir oxide at room temperature. After a $6 \mathrm{~h}$ stagnation period, the ambient solution was allowed to flow past the surface at $1 \mathrm{~s}^{-1}$ : 0 , region $1 ;[]$, region 2 . 
vibrational modes in region 2 became reoriented, with the backbone of the chain, in the direction of the flow. This preferred direction corresponds to the most intense component of the evanescent wave, thus enhancing the intensity of these vibrational modes. This effect occurred at the surprisingly low shear rate of $1 \mathrm{~s}^{-1}$. Although many other experiments agree that the effective hydrodynamic thickness responds to flow only at larger shear rates, in excess of $100 \mathrm{~s}^{-1}$, it has been argued that orientational disturbances must occur already at lesser flow rates [5].

This interpretation is supported by the data presented in Fig. 2, where we have plotted the oscillator strength in region 2 versus elapsed time for PI samples of four different molecular weights. These experiments suggest a systematic increase in susceptibility to orientation with increasing molecular weight. Similar trends were described by Lee and Fuller [6] and Chin and Hoagland [8], whose measurements of the effective hydrodynamic thickness indicated a greater decrease of thickness with increasing molecular weight.

Consider the later stages of the experiment depicted in Fig. 1. After an elapsed time of $19 \mathrm{~h}$, the flow of solution past the adsorbed layer was halted. The intention was to observe the relaxation as the chains in the layer found their way back to the conformation in the unperturbed quiescent

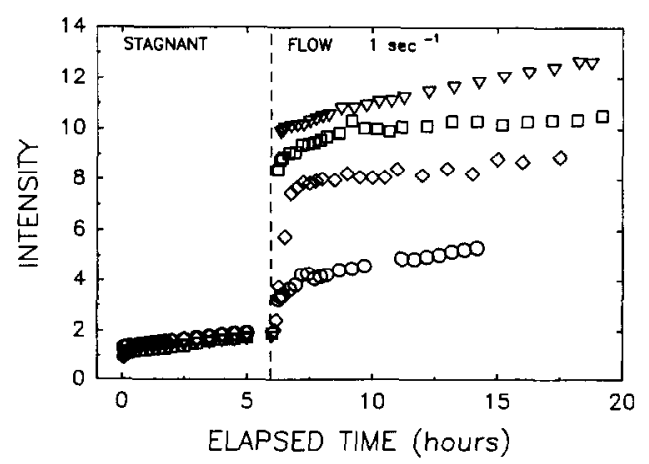

Fig. 2. Integrated intensity of polyisoprene of various molecular weights: $\left(0, M_{\mathrm{w}}=60000 ; \diamond, M_{\mathrm{w}}=135000 ; \square, M_{\mathrm{w}}=460000\right.$; $\nabla, M_{\mathrm{w}}=1600000$ ) plotted against elapsed time during adsorption from carbon tetrachloride solution (about $1 \mathrm{mg} \mathrm{ml}^{-1}$ ) onto initially bare Amtir oxide at room temperature. state. Although relaxation data in this regime showed poor quantitative reproducibility, the same trend was also observed in similar experiments with poly(methyl methacrylate) (PMMA). It is evident that relaxation after cessation of flow was very slow, far slower than the rate of orientation when flow was first initiated. This suggests some form of surface attachment or confinement of oriented segments, possibly associated with formation of additional trains at the solid surface, which impeded later relaxation.

The final experimental result worth mentioning here is presented in Fig. 3, where we have again plotted integrated intensity versus elapsed time. After the stagnation period, the shear rate was incrementally increased, every hour, from 0.4 to $1.6 \mathrm{~s}^{-1}$. Within this narrow range no dependence on shear rate was observed. It appears that over the range of shear rates studied, some maximum amount of potential alignment was reached at a low shear rate.

The magnitude of oscillator augmentation described here raises questions about the conformation in the quiescent state. If initially the segments were oriented isotropically, the augmentation could be no more than threefold [23], which implies considerable segmental orientation of adsorbed polymers even in the quiescent state. Such orientation during adsorption in the quies-

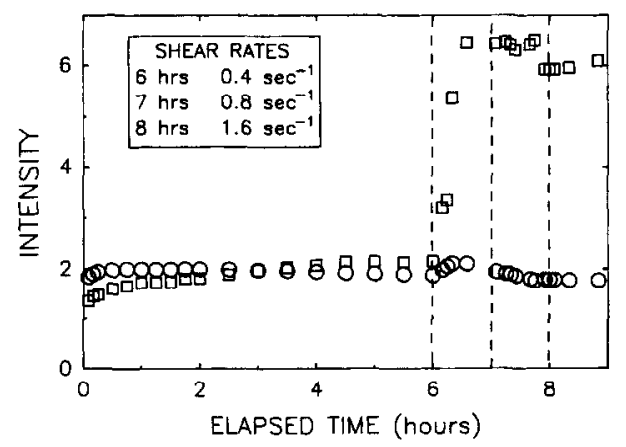

Fig. 3. Integrated intensity of polyisoprene $\left(M_{\mathrm{w}}=135000\right)$ plotted against elapsed time during adsorption from carbon tetrachloride solution (about $1 \mathrm{mg} \mathrm{ml}^{-1}$ ) onto initially bare Amtir oxide at room temperature (flow rates of $0.4 \mathrm{~s}^{-1}, 0.8 \mathrm{~s}^{-1}$, and $1.6 \mathrm{~s}^{-1}$ were sequentially implemented after a $6 \mathrm{~h}$ stagnation period): $\bigcirc$, region 1 ; $\square$, region 2 . 
cent state has been reported by Van Alsten [20]. However, a present limitation of the experiment is the poorly characterized polarization of the electric field [23] at the surface of the cylindrical ATR crystal used in this study. While these uncertainties preclude a quantitative interpretation of the extent of segmental orientation at this point, we have also obtained qualitatively similar results in experiments using PMMA. We believe that the trends described above are clear and convincing.

\section{Acknowledgement}

We acknowledge support through the National Science Foundation (Polymers Program), grant NSF-DMR-91-01509.

\section{References}

1 M.A. Cohen Stuart, T. Cosgrove and B. Vincent, Adv. Colloid Interface Sci., 24 (1986) 143.

2 P.-G. DeGennes, Adv. Colloid Interface Sci., 27 (1987) 189.

3 M. Kawaguchi and A. Takahashi, Adv. Colloid Interface Sci., 37 (1992) 219.

4 S. Granick, in I. Sanchez (Ed.), Physics of Polymer Surfaces and Interfaces, Manning Publications Co., Greenwich, CT, 1992.

5 J.-J. Lee and G.G. Fuller, J. Colloid Interface Sci., 103 (1984) 569 .

6 J.-J. Lee and G.G. Fuller, Macromolecules, 17 (1981) 375

7 G.J. Besio, R.K. Prud'homme and J.B. Benziger. Macromolecules, 21 (1988) 1070.

8 S. Chin and D.A. Iloagland, Macromolecules, 24 (1991) 1876.

9 R.S. Parnas and Y. Cohen, Macromolecules, 24 (1991) 4646.

10 Y. Cohen, Macromolecules, 21 (1988) 494.

11 Ph. Gramain and Ph. Myard, Macromolecules. 14 (1981) 180.

12 M. Bagassi, G. Chauveteau. J. Lecourtier, J. Englert and M. Tirrell, Macromolecules, 22 (1989) 262.

13 D.J. Kuzmenka and S. Granick, Colloids Surfaces, 31 (1988) 105

14 H.E. Johnson and S. Granick, Macromolecules, 23 (1990) 3367.

15 H.E. Johnson, J.F. Douglas and S. Granick, Phys. Rev. Lett., 326770 (1993).

16 M.A. Cohen Stuart, G.J. Fleer and J.M.H.M. Scheutjens, J. Colloid Interface Sci., 97 (1984) 515.

17 L.J. Bellamy, The Infrared Spectra of Complex Molecules. Wiley, New York, 1975.

18 R. Nuzzo, personal communication, 1993.

19 P.A. Flournoy and W.J. Schaffers, Spectrochim. Acta, 22 (1966) 5 .

20 J. Van Alsten, Macromolecules, 25 (1992) 3007.

21 C. Sung, Polym. Prepr., Am. Chem. Soc, 31 (1990) 521.

22 P. Frantz and S. Granick, Phys. Rev. Lett., 66 (1991) 899.

23 N.J. Harrick, Internal Reflection Spectroscopy. Interscience, New York, 1967. 